\title{
Galaxy Clusters and X-Ray Sources
}

$\mathrm{X}$-RAY astronomy continues to develop so rapidly that the wealth of available data is only beginning to be put into some sort of order by the theoreticians. Within the galaxy the range of sources covers types as diverse as the Crab Nebula, bright X-ray "novae" or flare stars, the recently detected X-ray pulsar in Cygnus and even the Sun. Perhaps this confusing flood of information is one of the factors which has encouraged some astronomers to turn their attention outside the galaxy, where experimental limitations provide a more stringent restriction on the number of phenomena which can be detected and thus make it easier to pick out patterns in the data.

Whatever the reason for their interest in extragalactic phenomena, the team of A. G. Cavaliere, H. Gursky and W. H. Tucker have indeed found some intriguing features in the data. These are described on page 437 of this issue of Nature, and pose as many questions as they answer, as is so often the case in this young branch of astronomy. Once again, the satellite Explorer 42 (UHURU) has been instrumental in providing the vital observations. In brief, the chief conclusion drawn from the observations of extragalactic X-ray sources is that there is a category of intense X-ray sources associated with clusters of galaxies, rather than with individual galaxies, or with quasars. This means that the X-ray producing region is not confined in a small volume of space--in the case of M 87 a region of the sky one degree across seems to be emitting around the prominent galaxy.

With the data so far available, it is not clear whether the regions of intense X-ray emission are at the central, dense parts of the galaxy clusters observed. Three sources of this new type of X-ray source do indeed seem to lie at the centres of galaxy clusters, and the very scarcity of data which makes it profitable for the theoretician to consider extragalactic phenomena ensures that, for the present at least, relatively simple theories explaining the phenomenon in terms of active dense central regions of galaxy clusters cannot be dismissed. The familiar idea that galaxies are born from an active region at the centre of a cluster is one that will certainly receive a stimulus from these observations.

But the other arguments are bound to be put forward by more conservative astronomers. The sources could arise simply from the superposition of X-radiation from many galaxies in the cluster, or this might come from one bright galaxy in each case. The latter possibility, however, seems rather dubious, particularly when it is seen that NGC 4847, the most likely candidate for such activity in the Coma cluster, would need to be emitting a degree of $\mathrm{X}$-radiation totally inconsistent with optical and radio observations. Again, the Coma cluster contains several galaxies showing activity in the form of jets and other features, so that the superposition model is reinforced.

In this context, it is particularly interesting that the current issue of Astrophysical Journal Letters contains two reports on the detection of yet another flaring X-ray source within the galaxy (166, L69 and L73 ; 1971). Both these reports, coming from groups working at the Massachusetts Institute of Technology, add weight to the growing conviction that there is enough intermittent strong $X$-ray activity in our galaxy to make it a respectable $\mathrm{X}$-ray source in its own right when viewed from outside.

Undoubtedly this accumulation of information will swing opinion in line with the view that almost all galaxies are X-ray sources, and that dense clusters must therefore naturally be expected to appear as one diffuse source of $\mathrm{X}$-radiation. But will the solution of the problem really be that easy? Cavaliere et al. point to other possible mechanisms, each of which fails to explain all the observed properties of these sources. If there is one thing which the explosion of high energy astronomy has emphasized, it is that there are no easy solutions to the problems confronting astronomers today.

\section{How Aspirin Functions}

THE vast consumption of aspirin in modern medical practice is a powerful indicator of the therapeutic value of this drug. In spite of suspicions that there may be circumstances in which the use of aspirin has untoward consequences, it remains one of the safest and most valuable of drugs. It is therefore a great puzzle that so little has been known about the method of action of aspirin and related materials with similar therapeutic effects. A part of the reason, of course, is that the therapeutic value of aspirin lies in the generality of its effects. Not merely is it an analgesic but valuable in the treatment of inflammation of various kinds as well as the treatment of fever. With such a simple chemical structure, it is natural to look for some highly specific chemical interaction, but with such generalized therapeutic consequences, it is clear that this specific biochemical interaction must be widely distributed throughout the body. But where do these specific interactions take place?

Only in the past few years or so has it become apparent that the substances the biochemical function of which may be controlled by aspirin can indeed be isolated and studied. In 1969, Piper and Vane (Nature, 223, $29 ; 1969$ ) demonstrated that inflammatory conditions in, for example, the lungs of guinea-pigs release materials which are themselves capable of stimulating the contraction of smooth muscles in isolated preparations. It was also clear that the same process was inhibited by aspirin and similar drugs. Inevitably, as it may seem, it has since turned out that the then unidentified substances are the ubiquitous prostaglandins. In short, there has been a growing suspicion that aspirin and related drugs may be therapeutically effective because of some relationship with the prostaglandins.

In next Wednesday's issue of Nature New Biology this tale is carried a big step further. In a group of three 